\title{
Levantamento de moscas-das-frutas e de seus parasitóides no município de Ferreira Gomes, Estado do Amapá
}

\author{
Survey of fruit flies and its parasitoids in Ferreira Gomes, Amapá State
}

Wilson Rodrigues da Silva ${ }^{\mathrm{I}}$ Ricardo Adaime da Silva ${ }^{\mathrm{I} *}$

\section{RESUMO}

Este trabalho objetivou efetuar um levantamento das espécies de moscas-das-frutas e de seus parasitóides, no município de Ferreira Gomes, Estado do Amapá. Entre os meses de março e julho de 2005, foram coletadas 38 amostras de frutos de seis espécies vegetais, totalizando $43,7 \mathrm{~kg}$. Foram obtidos 515 pupários, dos quais emergiram 123 adultos da família Tephritidae e 102 parasitóides. Foram obtidas cinco espécies de Anastrepha: A. fraterculus, A. obliqua, A. striata, A. antunesi e A. turpiniae. Os únicos hospedeiros de tefritídeos foram o taperebá (Spondias mombin) e a goiaba (Psidium guajava), com índices de infestação natural de 0,51 e 0,24 pupário/fruto e de 52,3 e 5,4 pupários $\mathrm{kg}^{-1}$ de fruto, respectivamente. As espécies dominantes foram A. obliqua, no taperebá, e A. striata, na goiaba. Foram obtidos exemplares de quatro espécies de Braconidae: Doryctobracon areolatus, Utetes anastrephae, Opius bellus e Opius sp., sendo a primeira a mais abundante.

Palavras-chave: Tephritidae, Braconidae, Amazônia, ocorrência, registro.
\end{abstract}

- NOTA -

\section{ABSTRACT}

This research was aimed at doing a survey on fruit flies and their parasitoids species in Ferreira Gomes, Amapá State, Brazil. Exactly 38 samples of fruits of six vegetables species were collected and it totaled $43,7 \mathrm{~kg}$ from March to July of 2005. It was possible to obtain 515 puparia that emerged 123 Tephritidae adults and 102 parasitoids. Moreover, five species of Anastrepha were registered: A. fraterculus, A. obliqua, A. striata, A. antunesi and A. turpiniae. Spondias mombin and Psidium guajava were the only hosts of Tephritidae species, they had indices of natural infestation of 0.51 e 0.24 puparia/ fruit and 52.3 and 5.4 puparia $\mathrm{kg}^{-1}$, respectively. The dominant species were A. obliqua, in S. mombin, and A. striata, in $\mathbf{P}$. guajava. Four species of Braconidae were registered: Doryctobracon areolatus, Utetes anastrephae, Opius bellus and Opius sp. Of all these species, the first one was the most abundant.

Key words: Tephritidae, Braconidae, Amazon, occurence, record.

Os levantamentos das espécies de moscasdas-frutas, de suas plantas hospedeiras e de seus parasitóides enquadram-se entre os estudos fundamentais para uma melhor compreensão desse grupo de insetos (ZUCCHI, 2000a). A correta identificação das espécies envolvidas é fundamental para o sucesso de programas de manejo integrado dessas pragas, visto que elas podem apresentar comportamentos distintos, exigindo estratégias específicas de manejo.

O conhecimento disponível sobre moscasdas-frutas no Estado do Amapá é muito limitado, tendo os primeiros resultados sido obtidos e divulgados a partir do ano de 1996, principalmente em função da detecção oficial da mosca-da-carambola, Bactrocera carambolae Drew \& Hancock, em março do mesmo ano, no município de Oiapoque. Atualmente, a praga concentra-se na região do referido município e, eventualmente, ocorre em outras localidades do Estado. Recentemente, foram intensificados os estudos com moscas-das-frutas, objetivando, de forma particular, a prospecção de hospedeiros da mosca-da-carambola,

IEmbrapa Amapá, Rodovia JK, km 5, 68903-000, Macapá, Amapá, Brasil. E-mail: adaime@cpafap.embrapa.br. *Autor para correspondência. 
visto que esta representa uma ameaça à fruticultura do Brasil, sendo caracterizada como praga quarentenária A2, ocorrendo exclusivamente no Estado do Amapá (SILVAet al., 2004).

Até o momento, foram registradas onze espécies do gênero Anastrepha no Estado do Amapá: A. antunesi Lima, A. coronilli Carrejo \& González, $\boldsymbol{A}$. distincta Greene, A. fraterculus (Wiedemann), $A$. leptozona Hendel, A. limae Stone, A. obliqua Macquart, A. serpentina (Wiedemann), A. striata Schiner, A. turpiniae Stone e A. zenildae Zucchi (RONCHI-TELES et al., 1996; RONCHI-TELES, 2000; SILVA\& RONCHI-TELES, 2000; CARVALHO, 2003; CREÃO, 2003; CREÃO \& RONCHI-TELES, 2004; URAMOTO et al., 2004; SILVA et al., 2005).

Em relação às espécies de parasitóides de tefritídeos no Estado do Amapá, GUIMARÃES et al. (2004) registraram Leptopilina boulardi (Barbotin, Carton \& Kelner-Pillaut). SILVA \& SILVA (2005) relacionaram outras sete espécies: Doryctobracon areolatus (Szépligeti), Doryctobracon sp., Opius sp., Opius bellus (Gahan), Utetes anastrephae (Viereck), Asobara anastrephae (Muesebeck) e Aganaspis pelleranoi (Brèthes). À exceção de $\boldsymbol{L}$. boulardi e $\boldsymbol{A}$. pelleranoi, que pertencem à família Figitidae, todos os demais insetos pertencem à Braconidae. Dentre as espécies citadas, destaca-se $\boldsymbol{D}$. areolatus, amplamente distribuído em vários municípios do Estado (SILVA \& SILVA, 2005).

Nos últimos anos, o Ministério da Agricultura, Pecuária e Abastecimento, por meio de sua Superintendência Federal de Agricultura no Amapá, tem realizado liberações de uma espécie de parasitóide exótico, Diachasmimorpha longicaudata (Ashmead), na tentativa de controlar a mosca-dacarambola (SILVA et al., 2004). A adaptação desta espécie na região de Oiapoque foi avaliada por CARVALHO (2003), após duas liberações de parasitóides, realizadas nos meses de fevereiro e abril do ano 2000. Pouco tempo após a primeira liberação, foram recuperados 42 exemplares de $\boldsymbol{D}$. longicaudata (em goiaba e taperebá) e obtidos 219 exemplares de espécies de parasitóides nativos (em abiu, taperebá e goiaba). Após a segunda liberação, foram recuperados 15 exemplares da espécie introduzida e obtidos 226 exemplares de espécies nativas, todos em goiaba.

O presente trabalho teve por objetivo realizar um levantamento de moscas-das-frutas e de seus parasitóides, no município de Ferreira Gomes, Estado do Amapá.

Foram realizadas amostragens de frutos, de março a julho de 2005, período em que se concentram as chuvas na região. As amostras foram coletadas ao acaso, localizando-se plantas com boa carga de frutos em maturação ou já maduros, apanhando-se frutos da planta e recém-caídos no solo. Os frutos foram acondicionados em sacos de algodão e levados para o Laboratório de Entomologia da Embrapa Amapá, onde foram contados, pesados e dispostos em bandejas de plástico, sobre uma camada de areia umedecida e esterilizada. As bandejas foram cobertas com tecido de organza, preso por ligas de borracha. A cada três dias, a areia foi examinada e os pupários retirados e transferidos para frascos de plástico transparente $(8 \mathrm{~cm}$ de diâmetro), com tampa vazada e coberta por organza, contendo uma fina camada de vermiculita umedecida. Os frascos foram dispostos em câmaras climatizadas, sob condições controladas de temperatura $\left(27 \pm 0,5^{\circ} \mathrm{C}\right)$, umidade relativa do ar ( $70 \pm 10 \%)$ e fotofase (12 horas), sendo observados diariamente.

As moscas-das-frutas e os parasitóides que emergiram foram acondicionados em recipientes de vidro, contendo álcool a 70\%, sendo posteriormente identificados no Laboratório de Entomologia da Embrapa Amapá, com base na literatura específica. Para as espécies de Anastrepha, os acúleos das fêmeas foram extrovertidos e examinados ao microscópio óptico (40x), conforme ZUCCHI (2000b).

Foram coletadas 38 amostras, que somaram 1.553 frutos, de seis espécies frutíferas, totalizando $43,7 \mathrm{~kg}$, de onde foram obtidos 515 pupários, com emergência de 43,7\% (moscas + parasitóides) (Tabela 1). A viabilidade pupal foi de 23,9\%, emergindo 123 moscas-das-frutas, todas do gênero Anastrepha, sendo 55 fêmeas e 68 machos (razão sexual de 44,7\%). Houve emergência de moscas somente de amostras de taperebá e goiaba (Tabela 1), que totalizaram 17,4 kg de frutos.

Os índices de infestação natural do taperebá e da goiaba foram 0,51 e 0,24 pupário/fruto e 52,3 e 5,4 pupários $\mathrm{kg}^{-1}$ de fruto, respectivamente. Foram identificadas cinco espécies de Anastrepha: $\boldsymbol{A}$. fraterculus, A. obliqua, A. striata, A. antunesi e A. turpiniae.

Das oito amostras de taperebá, sete estavam infestadas, dando origem a 41 fêmeas de $\boldsymbol{A}$. obliqua e quatro de A. antunesi. Das dez amostras de goiaba, três estavam infestadas, originando sete fêmeas de $\boldsymbol{A}$. striata, além de um exemplar de $\boldsymbol{A}$. fraterculus, $\boldsymbol{A}$. obliqua e A. turpiniae. Considerando os dois hospedeiros, a espécie mais abundante foi $\boldsymbol{A}$. obliqua (76,4\%).

No Estado do Amapá, A. obliqua já havia sido registrada em taperebá; $\boldsymbol{A}$. striata em goiaba, abiu, graviola, laranja, araçá-pêra, ingá-cipó e taperebá; $\boldsymbol{A}$. 
Tabela 1 - Famílias/espécies de frutos coletados, características das amostras e número de pupários e adultos das famílias Tephritidae e Braconidae emergidos. Ferreira Gomes, Amapá, 2005.

\begin{tabular}{|c|c|c|c|c|c|c|c|}
\hline \multirow[b]{2}{*}{ Famílias/Espécies } & \multicolumn{4}{|c|}{ Amostras } & \multirow{2}{*}{$\begin{array}{l}\text { Pupários } \\
\text { (n) }\end{array}$} & \multicolumn{2}{|c|}{ Exemplares emergidos } \\
\hline & $\begin{array}{l}\text { Quantidade } \\
\text { (n) }\end{array}$ & $\begin{array}{l}\text { Peso } \\
\text { (g) }\end{array}$ & $\begin{array}{l}\text { Frutos } \\
\text { (n) }\end{array}$ & $\begin{array}{l}\text { Infestadas } \\
\text { (n) }\end{array}$ & & $\begin{array}{l}\text { Tephritidae } \\
\text { (n) }\end{array}$ & $\begin{array}{l}\text { Braconidae } \\
\text { (n) }\end{array}$ \\
\hline $\begin{array}{l}\text { Anacardiaceae } \\
\text { Spondias mombin } \\
\text { (taperebá) }\end{array}$ & 08 & 8.985 & 924 & 7 & 470 & 103 & 102 \\
\hline $\begin{array}{l}\text { Myrtaceae } \\
\text { Psidium guajava (goiaba) }\end{array}$ & 10 & 8.399 & 189 & 3 & 45 & 20 & - \\
\hline $\begin{array}{l}\text { Passifloraceae } \\
\text { Passiflora edulis (maracujá) }\end{array}$ & 01 & 1.040 & 10 & 0 & 0 & - & - \\
\hline $\begin{array}{l}\text { Oxalidaceae } \\
\text { Averrhoa carambola } \\
\text { (carambola) }\end{array}$ & 09 & 15.552 & 158 & 0 & 0 & - & - \\
\hline $\begin{array}{l}\text { Myrtaceae } \\
\text { Syzygium jambos (jambo) }\end{array}$ & 05 & 7.154 & 110 & 0 & 0 & - & - \\
\hline $\begin{array}{l}\text { Combretaceae } \\
\text { Terminalia catappa } \\
\text { (castanhola) }\end{array}$ & 05 & 2.608 & 162 & 0 & 0 & - & - \\
\hline Total & 38 & 43.738 & 1.553 & 10 & 515 & 123 & 102 \\
\hline
\end{tabular}

antunesi em goiaba; A. turpiniae em goiaba e taperebá eA. leptozona em goiaba (RONCHI-TELES et al., 1996; CARVALHO, 2003; CREÃO, 2003; SILVA et al., 2005).

No presente trabalho, foram feitos novos registros de hospedeiros para A. obliqua (goiaba) e A. antunesi (taperebá). Além disso, esse trabalho apresenta o primeiro registro de frutos de goiaba como hospedeiros para $\boldsymbol{A}$. fraterculus, que anteriormente havia sido capturada em armadilhas McPhail por URAMOTO et al. (2004). Para o município de Ferreira Gomes, haviam sido registradas anteriormente $\boldsymbol{A}$. striata e A. leptozona, ambas em goiaba (CREÃO, 2003).

Foram obtidos 102 parasitóides em amostras de taperebá, caracterizando índice de parasitismo de 21,7\% (Tabela 1). Os exemplares pertencem a quatro espécies de Braconidae: D. areolatus, U. anastrephae, $\boldsymbol{O}$. bellus e Opius sp. Observou-se maior abundância de $\boldsymbol{D}$. areolatus (50,0\%), seguida de $\boldsymbol{O}$. bellus (36,3\%), Opius sp. (12,7\%) e U. anastrephae (1,00\%). Apenas a espécie $D$. areolatus havia sido registrada no município (CREÃO, 2003).

Embora algumas das outras espécies vegetais amostradas no presente trabalho sejam consideradas hospedeiros primários de Tephritidae (MALAVASI et al., 1980; ZUCCHI, 2000a), não apresentaram infestação.

\section{AGRADECIMENTO}

À Agência de Desenvolvimento da Amazônia, pelo suporte financeiro às pesquisas com moscas-das-frutas no Estado do Amapá.

\section{REFERÊNCIAS}

CARVALHO, R.S. Estudos de laboratório e de campo com o parasitóide exótico Diachasmimorpha longicaudata Ashmead (Hymenoptera: Braconidae) no Brasil. 2003. 182f. Tese (Doutorado em Biologia Genética) - Curso de Pósgraduação em Biologia Genética, Instituto de Biociências, Universidade de São Paulo.

CREÃO, M.I.P. Moscas-das-frutas (Diptera: Tephritidae): espécies, distribuição, medidas da fauna e seus parasitóides (Hymenoptera: Braconidae) no Estado do Amapá. 2003. 90f. Dissertação (Mestrado em Ciências Biológicas) - Programa Integrado de Pós-graduação em Biologia Tropical e Recursos Naturais, Instituto Nacional de Pesquisas da Amazônia, Universidade do Amazonas.

CREÃO, M.I.P.; RONCHI-TELES, B. Moscas-das-frutas (Diptera: Tephritidae): espécies, hospedeiros e distribuição geográfica no Estado do Amapá. In: CONGRESSO BRASILEIRO DE ENTOMOLOGIA, 20., 2004, Gramado, RS. Resumos... Gramado: SEB, 2004. 695p. p.658.

GUIMARÃES, J.A. et al. Levantamento e interações tritróficas de figitídeos (Hymenoptera: Eucoilinae) parasitóides de larvas frugívoras (Diptera) no Brasil. Arquivos do Instituto Biológico, São Paulo, v.71, n.1, p.51-56, 2004.

MALAVASI, A. et al. Biologia de "moscas-das-frutas" (Diptera, Tephritidae). I: lista de hospedeiros e ocorrência. Revista Brasileira de Biologia, Rio de Janeiro, v.40, n.1, p.9-16, 1980.

RONCHI-TELES, B. Ocorrência e flutuação populacional de espécies de moscas-das-frutas e parasitóides com ênfase para o gênero Anastrepha (Diptera: Tephritidae) na Amazônia brasileira. 2000. 156f. Tese (Doutorado em Ciências Biológicas) - Programa Integrado de Pós-graduação em Biologia Tropical e Recursos Naturais, Instituto Nacional de Pesquisas da Amazônia, Universidade do Amazonas. 
RONCHI-TELES, B. et al. New records of Anastrepha spp. (Dip., Tephritidae) and their hosts in Rondônia and Amapá States - Brazilian Amazônia. In: MEETING OF THE WORKING GROUP ON FRUIT FLIES OF THE WESTERN HEMISPhere, 2., 1996, Viña del Mar, Chile. Proceedings... Viña del Mar: s.ed., 1996. V.1, p.32-33.

SILVA, N.M.; RONCHI-TELLES, B. Amapá, Amazonas, Pará, Rondônia e Roraima. In: MALAVASI, A.; ZUCCHI, R. A. (ed.). Moscas-das-frutas de importância econômica no Brasil: conhecimento básico e aplicado. São Paulo: Holos, 2000. Cap.27, p.203-209.

SILVA, R.A. et al. Mosca-da-carambola: uma ameaça à fruticultura brasileira. Macapá: Embrapa Amapá, 2004. 15p. (Embrapa Amapá. Circular Técnica, 31).

SILVA, R.A.; SILVA, W.R. Conhecimento atual sobre parasitóides de moscas-das-frutas no Estado do Amapá. Macapá: Embrapa, 2005. 23p. (Embrapa Amapá. Documentos, 55).
SILVA, W.R. et al. Levantamento de moscas-das-frutas (Diptera: Tephritidae) na Área de Proteção Ambiental do Rio Curiaú, Macapá-AP. In: COLÓQUIO DE INICIAÇÃO CIENTÍFICA, 5., 2005, Macapá-AP. Anais... Macapá-AP: Seama, 2005. 1 CD-ROM.

URAMOTO, K. et al. Moscas-das-frutas (Diptera, Tephritidae) no Suriname e no Estado do Amapá, Brasil. In: CONGRESSO BRASILEIRO DE ENTOMOLOGIA, 20., 2004, Gramado, RS. Resumos... Gramado: SEB, 2004. 695p. p.668.

ZUCCHI, R.A. Espécies de Anastrepha, sinonímias, plantas hospedeiras e parasitóides. In: MALAVASI, A.; ZUCCHI, R.A. (Ed). Moscas-das-frutas de importância econômica no Brasil: conhecimento básico e aplicado. São Paulo: Holos, 2000a. Cap.4, p.41-48.

ZUCCHI, R.A. Taxonomia. In: MALAVASI, A.; ZUCCHI, R.A. (Ed.). Moscas-das-frutas de importância econômica no Brasil: conhecimento básico e aplicado. São Paulo: Holos, 2000b. Cap.1, p.13-24b. 